\title{
Thermal Inflation and the Moduli Problem
}

\author{
David H. Lyth \\ School of Physics and Chemistry, Lancaster University, Lancaster, LA1 4YB, U.K. \\ Ewan D. Stewart \\ Research Center for the Early Universe, School of Science, University of Tokyo, Bunkyo-ku, Tokyo 113, Japan
}

(September 1995)

\begin{abstract}
In supersymmetric theories a field can develop a vacuum expectation value $M \gg 10^{3} \mathrm{GeV}$, even though its mass $m$ is of order $10^{2}$ to $10^{3} \mathrm{GeV}$. The finite temperature in the early Universe can hold such a field at zero, corresponding to a false vacuum with energy density $V_{0} \sim m^{2} M^{2}$. When the temperature falls below $V_{0}^{1 / 4}$, the thermal energy density becomes negligible and an era of thermal inflation begins. It ends when the field rolls away from zero at a temperature of order $m$, corresponding to of order $10 e$-folds of inflation which does not affect the density perturbation generated during ordinary inflation. Thermal inflation can solve the Polonyi/moduli problem if $M$ is within one or two orders of magnitude of $10^{12} \mathrm{GeV}$.
\end{abstract}

\section{INTRODUCTION}

There is at present a 'standard model' of the Universe before nucleosynthesis, which is described in many reviews and several textbooks. According to this model, an early era of inflation sets the initial conditions for a Hot Big Bang, which starts far above the critical temperature for the electroweak transition $(T \simeq 100 \mathrm{GeV})$ and continues without interruption until the present matter dominated era begins.

This picture is pleasingly simple, but it is by no means mandatory in the context of current thinking about the fundamental interactions beyond the Standard Model. To be precise, it will not be valid if one or more scalar fields have a sufficiently large vev (vacuum expectation value) while at the same time having an almost flat potential. The reason is that the particle species corresponding to the oscillation around such a vev is typically both abundant and long lived, which modifies the simple picture in a significant and sometimes disasterous way. Extending an old terminology [1], we shall call a scalar field with a large vev and a flat potential a 'flaton field', or simply a 'flaton'.]

Although flaton fields are by no means inevitable, they are natural in the context of modern particle theory and in our opinion their possible cosmological consequences should be taken very seriously. Some aspects of the cosmology of flaton fields are already well known [1,3 10], and in a recent note [11] we drew attention to a new feature which we termed 'thermal inflation'. The present paper, along with two more in preparation [12,13], aims to give a systematic account of the subject.

Let us begin by being more precise about what is meant by a 'large' vev, and a potential which is 'almost flat'. These terms are defined with respect to the energy scale $10^{2}$ to $10^{3} \mathrm{GeV}$, which is the scale of supersymmetry breaking as defined by the masses of the supersymmetric partners of known particles 114. The vev is defined as the position of the minimum of the potential, and a 'large' vev $M$ is one satisfying $M \gg 10^{3} \mathrm{GeV}$. An 'almost flat' potential $V$ is one whose curvature $\left|V^{\prime \prime}\right|^{1 / 2}$ is of order $10^{2}$ to $10^{3} \mathrm{GeV}$ (except near any points of inflexion) out to field values much bigger than $10^{3} \mathrm{GeV}$, and if the field has a large vev this is supposed to be true out to at least the vev. For an almost flat potential the particle mass $m$ is therefore of order $10^{2}$ to $10^{3} \mathrm{GeV}$. From now on we drop the qualifier 'almost', referring simply to a flat potential.

The most widely discussed flaton candidates are the moduli occurring in superstring theory. The potential of a modulus is indeed flat, and if its vev is nonzero it is typically of order the Planck scale $M_{\mathrm{Pl}}=(8 \pi G)^{-1 / 2}=$ $2.4 \times 10^{18} \mathrm{GeV}$. A modulus with such a vev ${ }^{2}$ is known to be fatal to the standard cosmology since the corresponding particles are very abundant and do not decay before nucleosynthesis [2, 16 20]. As we shall see, the failure to decay

\footnotetext{
${ }^{1}$ Note the etymology. The term 'flaton' refers to the flat potential, not to inflation. Conversely, the familiar word 'inflaton' refers to the field which is slowly rolling during inflation. We shall also use the term 'flaton' to denote the particle species corresponding to a flaton field.

${ }^{2} \mathrm{~A}$ field with these properties occurred in the first example 15 of a nonrenormalizable supersymmetry-breaking hidden sector, which contained a single complex field. It was called the Polonyi field, and the associated problem [2] was called the Polonyi problem. Most of what we say concerning the moduli applies to any species with these properties.
} 
before nucleosynthesis is likely to persist for any flaton with a vev exceeding $10^{14} \mathrm{GeV}$, making all such flatons fatal to the standard cosmology [7].

Moduli are by no means the only flaton candidates. On the contrary, any field (in the observable sector) with a vev much bigger than $10^{3} \mathrm{GeV}$ is likely to have a flat potential, and so to be a flaton. The reason, as we discuss in detail below, is that it is natural to construct all available mass scales from just the two basic scales $m$ and $M_{\mathrm{Pl}}$. Apart from the moduli, the most familiar examples of fields with nonzero vevs are those which are charged under a continuous symmetry, the vev then indicating a spontaneous breakdown of the symmetry. If the symmetry is local then the field is by definition a higgs field, and presumably the examples of this type occurring in nature (apart from the higgs fields breaking electroweak symmetry) are the higgs fields breaking the GUT symmetry, whose vevs are of order $10^{16} \mathrm{GeV}$. Alternatively the symmetry could be global, a likely candidate for this case being the Peccei-Quinn field with a vev perhaps of order $10^{11} \mathrm{GeV}$. On the other hand, it makes perfect sense for a field to have a nonzero vev even if it is not charged under any continuous symmetry. For example, a right-handed neutrino mass might be generated by a vev, without lepton number being a good symmetry [21, 13.

As mentioned already, moduli as well as any other flatons with a vev bigger than $10^{14} \mathrm{GeV}$ are fatal to the standard cosmology. How are we to solve this 'moduli problem' if it exists?

The usual recipe for getting rid of unwanted relics in cosmology is to invoke an early epoch of inflation, lasting at least 50 to 60 Hubble times or so. Such an era is also desirable for other reasons [22,23], one of which is that it can generate an adiabatic density perturbation of the right magnitude to explain the cosmic microwave background anisotropy and large scale structure. To do this the potential at the end of inflation must satisfy $V^{1 / 4} \lesssim 10^{16} \mathrm{GeV}$ [24], and the lowest value of $V^{1 / 4}$ that has been proposed in a plausible model is $V^{1 / 4} \sim 10^{12} \mathrm{GeV}$ [25],26].

Inflation at such a high scale does not solve the moduli problem, because although it sufficiently dilutes moduli present before inflation they are regenerated with an unacceptable abundance afterwards. We show in [11, and in much more detail below, that to avoid excessive regeneration one requires

$$
V^{\frac{1}{4}} \lesssim 10^{7} \text { to } 10^{8} \mathrm{GeV}\left(\frac{\mathrm{GeV}}{T_{\mathrm{R}}}\right)^{\frac{1}{4}}
$$

where $T_{\mathrm{R}}$ is the reheat temperature. An era of inflation at such a low energy scale seems impossible to realize in the context of sensible particle physics, if it is required also to produce the cosmological density perturbation. Randall and Thomas [18] therefore suggested that the density perturbation is produced by an era of inflation at the usual high energy scale, while a second era of inflation at a low energy scale solves the moduli problem. However, even without the constraint of producing the density perturbation it is difficult to construct a model of inflation giving a sufficiently low energy scale, within the usual paradigm where there is an inflaton field rolling slowly down the potential. The reason stems from the fact that a necessary condition for slow roll is that the inflaton mass (or more precisely the curvature $\left|V^{\prime \prime}\right|^{1 / 2}$ evaluated while the field is rolling) be much less than the Hubble parameter $H \simeq V^{1 / 2} / M_{\mathrm{Pl}}$. The bound displayed in Eq. (11) corresponds to a very low mass $\lesssim 10 \mathrm{MeV}$.

The central purpose of this paper is to explore the fact that a flaton field can lead to a completely different type of inflation, called thermal inflation [11], which can solve the moduli problem provided that the vev $M$ is within one or two orders of magnitude of $10^{12} \mathrm{GeV}$. During thermal inflation the flaton field is held at the origin by finite temperature effects so that no field is rolling. The potential during thermal inflation is the value $V_{0}$ of the flaton potential at the origin, which is of order $m^{2} M^{2}$. With $M \sim 10^{12} \mathrm{GeV}$ this gives $V_{0}^{1 / 4} \sim 10^{7} \mathrm{GeV}$ which can satisfy Eq. (1). Thermal inflation starts when the thermal energy density falls below $V_{0}$ which corresponds to a temperature roughly $V_{0}^{1 / 4}$, and it ends when the finite temperature becomes ineffective at a temperature of order $m$, so the number of $e$-folds is $\frac{1}{2} \ln (M / m) \sim 10$. It turns out that this can sufficiently dilute the moduli existing before thermal inflation (especially if reheating after thermal inflation is delayed) and it will not interfere with the density perturbation generated during ordinary inflation. There is also the intriguing possibility that two or more bouts of thermal inflation can occur in quick succession, allowing an even more efficient solution of the moduli problem.

The present paper and its two successors are complementary to recent papers by Dine, Randall and Thomas [20,27. The latter focus on fields with a flat potential but zero vev. These fields too are liable to be oscillating in the early Universe and if they carry nonzero lepton or baryon number they can lead to baryogenesis (the Affleck-Dine mechanism). However baryogenesis in this way works only if there is no thermal inflation, and that in turn is a viable possibility only if there is no moduli problem. The two sets of papers therefore represent mutually exclusive scenarios for the early Universe, and only time will tell which if either is correct.

The rest of this paper is divided into two main sections plus a concluding one. In Section 2 we study the effective potential expected for flatons, both in the early Universe and in the present era when it reduces to the ordinary low energy effective potential. Special attention is paid to the case of moduli, which is different from that of other flatons because the moduli potential vanishes if supersymmetry is unbroken. The flaton decay rate is also estimated. The 
reheat process for homogeneous flaton oscillations is considered, taking account of possible parametric resonance. In Section 3 a systematic account is given of the history of the Universe, assuming that thermal inflation occurs and that there is a moduli problem. The concluding section summarizes the results, and points to future directions of research.

\section{FLAT POTENTIALS AND FLATONS}

In a generic supersymmetric gauge theory there will be a large number of directions in the space of the complex scalar fields ${ }^{3}$ in which the potential $V$ is exactly flat, before supersymmetry breaking and non-renormalizable terms are taken into account. (This is true, for example, in the Minimal Supersymmetric Standard Model.) After these effects are taken into account the potential is still almost flat, in the sense that the energy scale $\left|V^{\prime \prime}\right|^{1 / 2}$ specifying the curvature of the potential is only of order $10^{2}$ to $10^{3} \mathrm{GeV}$, out to field values many orders of magnitude bigger than this scale. In this paper we are interested in flaton fields, which by definition correspond to flat directions with a nonzero vev. The central theme of this paper is that flaton fields are cosmologically significant, because they typically lead to thermal inflation, and because they in any case oscillate homogeneously until a relatively late epoch.

A field with a nonzero vev is by definition either a higgs field or a gauge singlet. We will focus on the latter case in this paper, since a straightforward interpretation of the data indicate that the vev of the GUT higgs field is of order $10^{16} \mathrm{GeV}$ which is too high to give viable thermal inflation. Note, though, that in some GUT models there are additional higgs fields with much smaller vevs [28].

The cosmology of a given flaton field is largely determined by the form of its effective potential. One needs to know both the low energy effective potential which is relevant at the present era, and the effective potential in the early Universe. Also, since the case of moduli is somewhat different from that of flatons in general we treat the moduli in a separate subsection after the general discussion.

\section{A. The low energy effective potential}

Consider a complex flaton field $\phi$. In the limit where the potential is absolutely flat there is a global $U(1)$ symmetry under the transformation $\phi \rightarrow e^{i \alpha} \phi$, with an arbitrary choice for the origin of $\phi$. In the full theory this symmetry may survive for one choice of the origin, at least to a good approximation, or it may be so badly broken as to be unrecognizable.

\section{Global $U(1)$ symmetry}

We begin by considering the case where the symmetry survives. Extensions of the Standard Model can indeed contain spontaneously broken global $U(1)$ symmetries, a well known example being the Peccei-Quinn symmetry associated with the axion 29,22,23,30, 31. We initially suppose that the $U(1)$ symmetry is exact. The potential then depends on $\phi$ only through $|\phi|$, and assuming an effective theory that is valid right up to the Planck scale, the potential in the flat direction is typically of the form

$$
V=V_{0}-m_{0}^{2}|\phi|^{2}+\sum_{n=1}^{\infty} \lambda_{n} M_{\mathrm{Pl}}^{-2 n}|\phi|^{2 n+4}
$$

The $|\phi|^{2}$ term comes from soft supersymmetry breaking, which means that $m_{0} \sim 10^{2}$ to $10^{3} \mathrm{GeV}$, and the higher order terms are non-renormalizable terms. The dimensionless couplings $\lambda_{n}$ are at most of order 1 , if the theory is indeed valid up to the Planck scale.

The crucial feature of this potential, which distinguishes it from the potential of a generic field and makes it flat, is the absence of a term $\lambda|\phi|^{4}$ with $\lambda \sim 1$. Such a term can be forbidden by discrete or continuous gauge symmetries, in combination with supersymmetry. Supersymmetry breaking then generates a $\lambda|\phi|^{4}$ term with a suppressed coupling

\footnotetext{
${ }^{3}$ Each scalar field is complex in supersymmetric theories because supersymmetry relates it to the two degrees of freedom associated with a left- or right-handed spin-half field. In this paper we are assuming that the fields are canonically normalized in the regime of interest. (One cannot in general canonically normalize the fields exactly over an extended region of field space.)
} 
$\lambda \sim\left(m_{0} / M_{\mathrm{Pl}}\right)^{2}$. Such a term is negligible for flaton fields which are not moduli and we have lost nothing by omitting it from Eq. (2). (The case of moduli will be discussed in a moment, and in more detail in Section 2.5.)

As the notation suggests, we have in mind the case where the mass-squared at the origin, $-m_{0}^{2}$, is negative. This means that the vev of $|\phi|$ does not vanish but rather has a value $M \gg m_{0}$. To estimate $M$, suppose first that all of the $\lambda$ 's are of the same order. Then as one increases $|\phi|$, the $|\phi|^{6}$ term comes in first, leading to $M=\left(3 \lambda_{1}\right)^{-1 / 4} m_{0}^{1 / 2} M_{\mathrm{Pl}}^{1 / 2} \sim$ $\lambda_{1}^{-1 / 4} \times 10^{10}$ to $10^{11} \mathrm{GeV}$. Now suppose instead that this term is negligible, so that the $|\phi|^{8}$ term comes in first. Then $M=\left(4 \lambda_{2}\right)^{-1 / 6} m_{0}^{1 / 3} M_{\mathrm{Pl}}^{2 / 3} \sim \lambda_{2}{ }^{-1 / 6} \times 10^{13} \mathrm{GeV}$. If more terms are absent the vev will be raised further so the predicted range is $M \gtrsim 10^{10} \mathrm{GeV}$. In the entire regime $|\phi| \lesssim M$ the curvature $\left|V^{\prime \prime}\right|^{1 / 2}$ of the potential is only of order $m_{0}$, which is of order $10^{2}$ to $10^{3} \mathrm{GeV}$. In particular the mass $m$ of the flaton particle is of this order, and from now on we shall generally use it instead of $m_{0}$ when writing down order of magnitude estimates. The requirement $V(M)=0$ gives $V_{0} \sim m^{2} M^{2}$, corresponding to

$$
\left(\frac{V_{0}^{1 / 4}}{10^{6} \mathrm{GeV}}\right) \sim\left(\frac{M}{10^{10} \mathrm{GeV}}\right)^{1 / 2}
$$

If the $n$th term dominates in Eq. (2), then

$$
\begin{aligned}
m^{2} & =2(n+1) m_{0}^{2} \\
M^{2 n+2} M_{\mathrm{Pl}}^{-2 n} & =\left[2(n+1)(n+2) \lambda_{n}\right]^{-1} m^{2} \\
V_{0} & =[2(n+2)]^{-1} m^{2} M^{2}
\end{aligned}
$$

Rather than the non-renormalizable terms being suppressed by the Planck scale, they might be generated by integrating out particles with GUT scale masses and so instead be suppressed by $M_{\mathrm{GUT}} \simeq 2 \times 10^{16} \mathrm{GeV}$. This would correspond to taking $\lambda_{n} \lesssim\left(M_{\mathrm{Pl}} / M_{\mathrm{GUT}}\right)^{2 n}$ and would give the somewhat looser lower bound

$$
M \gtrsim 10^{9} \mathrm{GeV}
$$

We noted a moment ago that in Eq. (2) the $|\phi|^{4}$ term has a coupling $\lambda \sim\left(m / M_{\mathrm{Pl}}\right)^{2}$ which is many orders of magnitude less than 1. It may happen that the same is true of one or more further terms. But for a flaton which is not a modulus one expects to find, at not too high order, a term whose coupling $\lambda_{n}$ is not many orders of magnitude less than 1. As a result, one expects the vev of flaton which is not a modulus to be several orders of magnitude below $M_{\mathrm{Pl}}$. By contrast one expects for a modulus that all couplings are strongly suppressed, because the potential of a modulus vanishes exactly when supersymmetry is unbroken. A natural order of magnitude for the couplings of a modulus is $\left(m / M_{\mathrm{Pl}}\right)^{2}$ making the vev of order $M_{\mathrm{Pl}}$, though there are other possibilities. We shall discuss moduli in more detail in Section 2.5.

The flat potential Eq. (2) is not at all what cosmologists generally assume when they consider spontaneous symmetry-breaking in the early Universe. Rather they assume, as for instance in the textbooks [22,23, 32] and the reviews [29,33] that the potential is like the Standard Model higgs' potential]

$$
V=\lambda\left(|\phi|^{2}-M^{2}\right)^{2}
$$

with $\lambda \sim 1$. For the Standard Model higgs, whose vev is of order $10^{2} \mathrm{GeV}$, this potential is indeed natural from the viewpoint of supergravity; it simply corresponds to a non-flat direction, in which there is a $\lambda|\phi|^{4}$ term. But when $M$ is much bigger than $m$ it becomes far less natural, and in our view Eq. (2) rather than Eq. (8) should be regarded as the 'default' case.

So far we have taken the $U(1)$ symmetry to be exact, so that the goldstone boson corresponding to the angular direction is massless. If the symmetry is broken the goldstone boson will acquire a mass. This mass is by definition much less than that of the flaton if the symmetry is only slightly broken. On the other hand, as we now discuss the symmetry may be strongly broken which means that the would-be goldstone boson becomes just another flaton particle.

\footnotetext{
${ }^{4}$ The mass-squared of the flaton particle is $\frac{1}{2} V^{\prime \prime}(M)$ because the canonically normalized complex field $|\phi|$ is related to the canonically normalized real flaton particle field $\delta \phi$ by $|\phi|=M+\delta \phi / \sqrt{2}$.

${ }^{5}$ In the case of the Standard Model $\phi$ is a doublet and the symmetry is $S U(2)$ but this is an irrelevant complication for our purpose.
} 
As a simple example, consider the superpotential $W=\left(\lambda / 4 M_{\mathrm{Pl}}\right) \phi^{4}$ with $\lambda \sim 1$. After supersymmetry breaking the corresponding potential is of the form

$$
\begin{aligned}
V(\phi) & =V_{0}-m_{0}^{2}|\phi|^{2}+\left(A W+B \phi \frac{\partial W}{\partial \phi}+\text { c.c. }\right)+\left|\frac{\partial W}{\partial \phi}\right|^{2} \\
& =V_{0}-m_{0}^{2}|\phi|^{2}+\left(\frac{\lambda C \phi^{4}}{M_{\mathrm{Pl}}}+\text { c.c. }\right)+\frac{|\lambda|^{2}|\phi|^{6}}{M_{\mathrm{Pl}}^{2}}
\end{aligned}
$$

with $m_{0}$ and the magnitudes of $A, B$ and $C$ all of order $10^{2}$ to $10^{3} \mathrm{GeV}$.

In this example $U(1)$ has been broken down to $Z_{4}$ (which leaves $\phi^{4}$ invariant), and there are four vacua each with the same vev $|\phi|=M \sim|\lambda|^{-1 / 2} m_{0}^{1 / 2} M_{\mathrm{Pl}}^{1 / 2}$. In a given vacuum there are now two particles with mass $10^{2}$ to $10^{3} \mathrm{GeV}$; one of them is the one corresponding to the radial oscillation that we considered before, and the other is the would-be goldstone boson corresponding to the angular oscillation. We shall generally refer to them both as flatons. Note that in the regime $|\phi| \ll M$ the $U(1)$ symmetry is approximately restored, since the term $-m_{0}^{2}|\phi|^{2}$ dominates.

The $Z_{4}$ symmetry surviving in this example has ensured that there are no linear terms in the expansion of $\phi$ about the origin, and this feature will become crucial when we consider the effective potential in the early Universe. Of course any $Z_{n}$ symmetry will do for this purpose, and it does not need to be exact.

In our discussion $-m_{0}^{2}$ has been taken to be negative. If it is positive the potential has a minimum at the origin. If this is also the position of the vev (ie., if it is the absolute minimum) then the field is not a flaton and does not concern us. It can however happen, as for instance in the model of [34], that the origin corresponds to a false vacuum, with higher order terms generating a large vev so that we are dealing with a flaton. Thermal inflation with such a flaton is viable only if tunneling to the true vacuum is rapid, which is typically not the case.

For simplicity we shall from now on make frequent use of the notation appropriate to the case where there is a $U(1)$, writing the potential as a function only of $|\phi|$ and using $m$ to denote the mass of the flaton particle.

\section{B. The flaton decay rate}

There is a general expectation that a flaton particle corresponding to oscillations around a vev $M$ will couple only weakly to particles with mass much less than $M$. In particular, one expects [1,5,6,6,8 11] that the flaton decay rate $\Gamma$ is at most of order $m^{3} / M^{2}$.

Consider first the decay into a pair of identical spin zero particles which correspond to a real field $\psi$, with the renormalizable effective interaction $\lambda|\phi|^{2} \psi^{2}$. Setting $|\phi|$ equal to its vev this interaction gives a contribution $2 \lambda M^{2}$ to the mass-squared $m_{\psi}^{2}$. Barring a precise cancellation 6 it follows that

$$
\lambda \lesssim \frac{1}{2}\left(\frac{m_{\psi}}{M}\right)^{2}
$$

where the right hand side is at most $\frac{1}{8}(m / M)^{2}$ or the decay would be forbidden by energy conservation. Substituting $|\phi|=M+\delta \phi / \sqrt{2}$, one finds that the flaton decay rate corresponding to this interaction is

$$
\Gamma=\frac{\lambda^{2}}{8 \pi}\left(\frac{M}{m}\right)^{2} m \sqrt{1-4 m_{\psi}^{2} / m^{2}}
$$

Maximizing this expression subject to the constraint Eq. (11) gives $\Gamma \lesssim 10^{-4} m^{3} / M^{2}$.

This effective interaction with a coupling of order $\lambda \sim(m / M)^{2}$ is quite natural. For instance an interaction $|\phi|^{2} X^{2}$ might give some field $X$ a mass of order $M$, and then an interaction $\psi^{2} X^{2}$ would generate it through the diagram with a single $X$ loop.

\footnotetext{
${ }^{6}$ It has been pointed out to us by G. Dvali that such a cancellation does occur in an SU(5) GUT where the doublet-triplet splitting problem is 'solved' by a fine tuned cancellation. In such a case the decay rate has the unsuppressed value $\Gamma \sim m$. When the problem is solved in a more acceptable way this need not be so, but we will not pursue the point here because our main focus is not on the GUT.
} 
For an effective interaction involving more powers of the fields and/or derivatives the arguments are generally less precise, but one expects suppression because such terms are non-renormalizable and therefore involve inverse powers of some scale $\tilde{M}$ which is presumably at least of order $M$. Consider for instance a term involving one power of $\phi$ and two of $\psi$, with $2 n$ derivatives. Its coefficient is expected to be at most of order $\lambda^{\prime} M^{-2 n}$ with $\lambda^{\prime} \sim M$, and since the energy of all particles is of order $m$ (in the $\phi$ rest frame) this gives the decay rate Eq. (12) with $\lambda \sim(m / M)^{2 n}$. For $n>1$ this is much smaller than the upper limit Eq. (11), but for $n=1$ it is bigger by a factor $\left(m / m_{\psi}\right)^{2}$ leading to $\Gamma \sim(8 \pi)^{-1} m^{3} / M^{2}$. On the basis of this discussion, we shall assume that

$$
\Gamma=10^{-2} \gamma m^{3} / M^{2}
$$

with $\gamma \lesssim 1$.

\section{The decay into goldstone bosons}

A definite example of a derivative coupling is provided by the decay of the 'radial' flaton into the 'angular' flaton, or goldstone boson. Near the vev, the canonically normalized radial field $s$ and angular field $a$ are defined by

$$
\phi=\left(\frac{s}{\sqrt{2}}+M\right) \exp (i a / \sqrt{2} M)
$$

Expanding the canonical kinetic term $\mathcal{L}_{\text {kin }}=\partial_{\mu} \phi^{*} \partial^{\mu} \phi$ to first order in $s$, one finds the canonical kinetic terms for $s$ and $a$ plus an interaction term

$$
\mathcal{L}_{\text {int }}=\frac{s}{\sqrt{2} M} \partial_{\mu} a \partial^{\mu} a
$$

The coefficient is of the advertised form $\lambda^{\prime} / M^{2}$, with $\lambda^{\prime}=M / \sqrt{2}$.

The goldstone bosons produced by this coupling can be cosmologically dangerous, because their interaction can be too weak to thermalize them. This will be discussed in connection with the axion in [12] (see also [31]).

\section{The flaton freeze-out temperature}

Though we have focussed on the decay rate, similar considerations apply to collision rates. The rates for collisions involving a flaton and other light particles are suppressed at energies well below $M$, and therefore the freeze-out temperature below which flaton particles cease to be in thermal equilibrium is very roughly of order $M$. Note that this applies only in the true vacuum, where the flaton field is oscillating about the vev.

\section{The effective potential in the early Universe}

In the early Universe, the interaction of a given field with other fields will alter the effective potential of that field, and in particular the effective flaton potential $V(\phi)$ will be altered.

We should first clarify what is meant by the 'effective potential $V(\phi)$ '. There is in reality a single effective potential $V(\phi, \psi, \ldots)$, which is a function of all the scalar fields. It is natural to define the effective potential of any individual field as the full potential with all other fields held at their vevs, and this is the definition that we had in mind for the low energy effective potential $V(\phi)$. However in the early Universe all sufficiently light scalar fields are significantly displaced from their vevs, either homogeneously in the manner we have been discussing for flatons, or inhomogeneously as for instance if the field is in thermal equilibrium. Instead of evaluating the full effective potential $V(\phi, \psi, \ldots)$ with the other fields at their vevs one should set them equal to their current time-averaged values, so that for instance a term $\psi^{2} \phi^{2}$ is replaced by $\left\langle\psi^{2}\right\rangle \phi^{2}$. In addition, the actual form of the full effective potential is affected by the presence of particles with nonzero spin and also by kinetic terms, so that the effective potential $V_{\text {early }}(\phi, \psi, \ldots)$ in the early Universe is different from the low energy effective potential $V_{\text {low }}(\phi, \psi, \ldots)$ which applies at present. For both of these reasons, the effective potential $V_{\text {early }}(\phi)$ in the early Universe is different from the low energy effective potential $V_{\text {low }}(\phi)$ which applies at present.

Although the form of the effective potential $V(\phi)$ changes with the history of the Universe, its gradient will always vanish at the origin provided that it is invariant under at least a $Z_{n}$ symmetry. This tends to be at least approximately true in simple models, and we shall take it for granted in what follows. Let us pause briefly though to see why such a 
symmetry is common. If the full potential $V(\phi, \psi, \ldots)$ is expanded as a power series in all of the fields each individual term will be invariant under one or more $Z_{n}$ symmetries unless it consists of just the first power of one field. For instance the term $\phi^{2} \psi^{2}$ is invariant under a $Z_{2}$ acting on $\phi$, and another acting on $\psi$. As we discussed in Section 2.1 , only a few leading terms will be important in practice, so it is reasonable that one or more $Z_{n}$ symmetries will be approximately present in the full potential. Then the question of whether or not the potential $V(\phi)$ of an individual field possesses an approximate $Z_{n}$ symmetry depends on the form of the full potential, but again this is not unreasonable.

Taking it for granted that the gradient of $V(\phi)$ vanishes at the origin, let us ask what is the effective mass-squared $V^{\prime \prime}(0)$ in the early Universe. (We continue to assume for simplicity that there is a $U(1)$ symmetry, so that $V$ is a function only of $|\phi|$.)

First consider the era of ordinary inflation. It has been known for some time [3, $4,8,26$ ] that by looking at the form of the full potential predicted by $N=1$ supergravity one can identify contributions of order $\pm H^{2}$ to the mass-squared of every field. For the inflaton field(s) these contributions have to cancel because otherwise inflation will not occur, but for a generic field one does not expect a cancellation. Assuming that flatons are not inflatons, the conclusion is that their mass-squared during inflation is (at least) of order $\pm H^{2}$.

After inflation it is not so clear what the mass-squared will be. In the extreme case where the interaction is of only gravitational strength one expects a contribution of the same order, $\pm H^{2}$ [20]. We noted earlier that in the true vacuum, the interaction of flaton particles with other light particles is suppressed, so one at first sight expects something like this estimate to hold for a flaton field. However, that suppression occurs because the vev of the flaton field is large (the flaton particles correspond to small oscillations around the vev). Near the origin the flaton field can have unsuppressed interactions with light fields.

To see why, take as an example the interaction $\frac{1}{2} \lambda|\phi|^{2} \psi^{2}$ that we considered earlier. When $\phi$ is at its vev this gives a contribution $\lambda M^{2}$ to $m_{\psi}^{2}$. Barring cancellations, $\lambda$ must therefore be small if $m_{\psi}$ is small. But suppose that in contrast $m_{\psi}$ is of order $M$ and is generated by this interaction. Then there is a coupling $\lambda \sim 1$, and for flaton field values near the origin the $\psi$ field becomes light. The result is that near the origin the flaton field has an unsuppressed interaction with the light field $\psi$.

If $\phi$ is a higgs field, charged by definition under a gauge symmetry, a coupling of this kind to at least the gauge bosons and gauginos is inevitable. In the case where $\phi$ is neutral under all gauge symmetries, which is our focus here, such a coupling is not inevitable but it is still quite natural; for instance, in models of the kind discussed in [21, 13, 12. a flaton field couples in this way to the right handed neutrino and sneutrino.

Assuming that the flaton field near the origin indeed has unsuppressed interactions with one or more particle species having effective mass of order $|\phi|$, it will be in thermal equilibrium in the regime $|\phi| \lesssim T$. (The upper limit comes from the fact that at a given temperature particles with mass bigger than $T$ become too rare too maintain thermal equilibrium.) The finite temperature correction to the effective potential gives the flaton an effective mass-squared [1.35] of order $\left(T^{2}-m_{0}^{2}\right)$, which gives the effective potential a local minimum at the origin for $T$ bigger than some critical temperature $T_{\mathrm{C}} \sim m_{0} \sim m$. (As usual, $-m_{0}^{2}$ denotes the effective zero-temperature mass-squared at the origin, and $m$ denotes the flaton particle mass which is the parameter we normally focus on. Recall that both $m_{0}$ and $m$ are of order $10^{2}$ to $10^{3} \mathrm{GeV}$.)

In addition to the local minimum at the origin, the effective potential retains its true minimum at $\phi=M$ except at very high temperatures $T \gtrsim M$, but there is no significant tunneling between the two [1. [55].

To summarize this discussion, if the flaton field has gravitational strength interactions its mass-squared is expected to be of order $\pm H^{2}$. If, on the other hand, it has unsuppressed interactions then it will be in thermal equilibrium in the regime $|\phi| \lesssim T$ and in this regime there will be a necessarily positive mass-squared of order $T^{2} \sim\left(M_{\mathrm{Pl}} / H\right) H^{2}$. These are the most important possibilities for the effective mass-squared but others exist, especially during inflation where one might have a coupling to the inflaton field, say of the form $\psi^{2} \phi_{\text {inf }}^{2}$ (in particular, hybrid inflation [25] makes essential use of such a coupling). As in this example, the positivity of the potential tends to require that such a coupling again gives a positive mass-squared.

\section{The cosmology of fields with flat potentials}

In the light of what we have done so far there are the following four possibilities for the cosmology of a field with a flat potential.

(i) The field sits at the origin. If the minimum of the potential is at the origin throughout the history of the Universe then the field will sit there apart from thermal and quantum fluctuations. In that case it does not undergo homogeneous oscillations in the early Universe, and we are not concerned with it here. It will in general have 
unsuppressed interactions (at least if it is not a modulus) and the corresponding particle species will be produced through particle collisions and decays involving these interactions.

(ii) The field oscillates about the origin. Now suppose that although the minimum of the low energy effective potential is at the origin, the minimum in the early Universe is displaced because there is a negative mass-squared of order $-H^{2}$. In that case the field will start to oscillate about the origin at the epoch $H \sim m$. The oscillation is generally short lived, because the particles corresponding to it generally have unsuppressed couplings (except perhaps for moduli). If there is no thermal inflation the oscillation can however lead to viable baryogenesis through the Affleck-Dine mechanism [27].

(iii) Thermal inflation occurs. In the two remaining cases the vev is nonzero, so that we are dealing by definition with a flaton field. Thermal inflation, which is the focus of the present paper, occurs if the flaton field is held at zero in the early Universe by the finite temperature. It ends when the temperature falls to some critical value $T_{\mathrm{C}} \sim m$ (provided that the zero-temperature effective potential has no barrier separating the origin from the vev), after which the flaton field starts to oscillate about the vev. The oscillation around the vev might persist for a long time because the coupling of flaton particles to other light particles is suppressed (Section 2.2, and Section 2.6 below).

(iv) Flatons not leading to thermal inflation. In the fourth case the flaton field fails to be held at the origin by the finite temperature of the early Universe. This will occur if the flaton has an effective mass-squared $\sim-H^{2}$ which prevents it from ever being near the origin. It will also occur whatever the sign of the mass-squared, if the interaction of the flaton field is suppressed even near the origin. When $H$ falls to a value of order the flaton mass $m$, the field starts to oscillate about the vev, with an initial amplitude of order $M$. (The initial amplitude is equal to $M$ if the initial field value is at the origin. If the field is displaced from the origin by a mass-squared of order $-H^{2}$, its value is typically of order $M$ when the field starts to oscillate.) As in the previous case the oscillation might last for a long time.

\section{E. The moduli potential}

What we have done so far, including the summary of the last subsection, applies in essence to all flaton fields including any which are moduli. On the other hand, moduli do have some properties which distinguish them from other scalar fields ('matter fields') and as a result the general discussion acquires a somewhat different flavour when applied to them.

The low energy effective potential of a modulus vanishes exactly if supersymmetry is unbroken. After supersymmetry breaking its potential is generally thought to be flat, so that its curvature $\left|V^{\prime \prime}\right|^{1 / 2}$ is everywhere of order $10^{2}$ to $10^{3} \mathrm{GeV}$ (except near points of inflexion). If a modulus has a nonzero vev, then as we discuss in a moment its vev is generally expected to be of order $M_{\mathrm{Pl}}$. To a large extent its properties can then be obtained simply by setting $M=M_{\mathrm{Pl}}$ in formulas that apply to flatons in general, but there are some special features. These arise because one is forced to consider field variations of order $M_{\mathrm{Pl}}$, in contrast with matter fields where one need only consider much smaller variations (typically of order the vev $M \ll M_{\mathrm{Pl}}$ for a flaton field which is not a modulus).

In order to talk about a nonzero vev for any field there has to be a well defined origin, which will be defined as a point which is invariant ('fixed') under the group of symmetries under which the field transforms. For matter fields this defines a unique origin, such that the symmetry group consists of linear operators in field space. For moduli the symmetries are more complicated, and there are in general an infinite number of fixed points with a separation of order $M_{\mathrm{Pl}}$ (though only a finite number are physically distinct because the symmetry is a discrete gauge symmetry). If the vev of a modulus is at a fixed point it is natural to say that it vanishes, and otherwise it is natural to define the vev as the distance to the nearest fixed point. These are the conventions that we have had in mind, without explicitly stating them. The statement that the vev of some modulus is of order $M_{\mathrm{Pl}}$ just means that it is not close to any particular fixed point. As with other fields, a modulus can have unsuppressed interactions with other light fields only if it is close to a fixed point.

Each of the four possibilities for the cosmology of a flaton field listed in the last subsection exists for a modulus. If possibility (i) holds for all moduli then there is no moduli problem. Assuming that this is not the case, let us look at the expected form of the effective potential of a modulus $\Phi$. For simplicity we will pretend that $\Phi$ is real, and take it to be canonically normalized. Before supersymmetry breaking is taken into account the potential $V(\Phi)$ vanishes. With the breaking taken into account the potential in the true vacuum (the low energy potential) is generally thought to be of the form

$$
V_{\text {true }}=M_{\mathrm{S}}^{4} f\left(\frac{\Phi}{M_{\mathrm{Pl}}}\right)=\frac{1}{2} m_{\Phi}^{2}\left(\Phi-\Phi_{1}\right)^{2}+\ldots
$$

Here the supersymmetry breaking scale $M_{\mathrm{S}}$ is related to the scale $m \sim 10^{2}$ to $10^{3} \mathrm{GeV}$ by $M_{\mathrm{S}} \sim\left(m M_{\mathrm{Pl}}\right)^{1 / 2} \sim 10^{10}$ to $10^{11} \mathrm{GeV}$, and $f(x)$ is a function whose value and low order derivatives are typically of order 1 in the regime $|x| \lesssim 1$. 
We have expanded the potential about its vev $\Phi_{1}$. Note that the potential vanishes in the limit $M_{\mathrm{S}} \rightarrow 0$ of unbroken supersymmetry, in accordance with the fact that we are dealing with a modulus.

In the early Universe there will be additional supersymmetry breaking because of the nonzero energy density $\rho$, leading to an additional contribution to the potential of the form [3, [, 26, 20]

$$
V_{\text {cosm }}=\rho g\left(\frac{\Phi}{M_{\mathrm{Pl}}}\right)=\frac{\alpha}{2} H^{2}\left(\Phi-\Phi_{2}\right)^{2}+\ldots
$$

The function $g(x)$ has value and low order derivatives of order 1 (making $\alpha \sim 1$ ). The minimum of this potential is located at a different value $\Phi_{2}$, which is displaced from the true vev $\Phi_{1}$ by a distance $\Phi_{0}=\Phi_{2}-\Phi_{1} \sim M_{\mathrm{Pl}}$.

We have in mind the case where both $\Phi_{1}$ and $\Phi_{2}$ are nonzero (case (iv) of the last subsection) and of order $M_{\mathrm{Pl}}$. If $\Phi_{2}=0$ but $\Phi_{1} \neq 0$ (case (iii)) there is also a moduli problem, but it might be rendered insoluble by domain walls (though in analyzing this possibility within a given model one will have to remember that the discrete symmetries under which the moduli transform are gauge symmetries). If $\Phi_{1}=0$ but $\Phi_{2} \neq 0$ (case (ii)) there is no moduli problem if the relevant moduli have unsuppressed couplings near the origin.

Although Eq. (16) is the simplest possibility for the potential of a modulus there are others, which could lead to a vev below the Planck scale. For example, if supersymmetry breaking is due to hidden sector gaugino condensation then the moduli potential might include terms of the form $\mu^{n}|\phi|^{m+4} / M_{\mathrm{Pl}}^{n+m}$ where $\mu$ is related to vevs arising from gaugino condensation. These terms still vanish when supersymmetry is unbroken, as is required for a modulus, but they might generate a vev below the Planck scale. For example the GUT Higgs could be a Wilson line modulus, with a vev of order $10^{16} \mathrm{GeV}$ generated in this way [36]. In considering the moduli problem we assume in this paper that at least some moduli have a vev of order $M_{\mathrm{Pl}}$.

\section{F. The flaton reheat temperature}

Let us quantify the statement that the flaton field oscillations in the early Universe last for a long time.

The oscillation of a flaton field with vev $M$ has initial amplitude $\phi_{0} \sim M$. The corresponding energy density is $\rho_{\phi} \sim \frac{1}{2} m^{2} \phi_{0}^{2}$, and the number density of the flaton particles is $n_{\phi} \sim \frac{1}{2} m \phi_{0}^{2}$. These particles have no random motion because the field is homogeneous, so they constitute matter as opposed to radiation. If the flaton is associated with thermal inflation, the oscillation commences after thermal inflation and immediately dominates the energy density. If not, the oscillation commences at the earlier epoch $H \sim m$, and may or may not come to dominate the energy density.

If the oscillation amplitude decreased like $a^{-3 / 2}$, where $a$ is the scale factor of the Universe, then the energy per comoving volume of the flaton field would be conserved. In fact, the energy drains away through the interactions of the flaton field so that the oscillation amplitude decreases faster.

If the oscillation amplitude is sufficiently small and the interactions are sufficiently weak, each flaton particle decays independently so that the rate at which the energy drains away is simply the particle decay rate $\Gamma$. It has practically all disappeared soon after the time

$$
\Gamma^{-1} \simeq 3 \gamma^{-1} 10^{-9}\left(\frac{M}{10^{11} \mathrm{GeV}}\right)^{2}\left(\frac{300 \mathrm{GeV}}{m}\right)^{3} \operatorname{secs}
$$

where we have used Eq. (13). Setting this time equal to $H^{-1}$ and assuming that the decay products thermalize promptly we arrive at an estimate of the 'reheat temperature',

$$
T_{\mathrm{D}} \simeq g_{*}^{-\frac{1}{4}} \Gamma^{\frac{1}{2}} M_{\mathrm{Pl}}^{\frac{1}{2}} \simeq 3 \gamma^{\frac{1}{2}}\left(\frac{10^{11} \mathrm{GeV}}{M}\right)\left(\frac{m}{300 \mathrm{GeV}}\right)^{\frac{3}{2}} \mathrm{GeV}
$$

where $g_{*} \sim 10^{2}$ is the effective number of species at $T=T_{\mathrm{D}} \cdot 7$

\footnotetext{
${ }^{7}$ The following results will be used without comment in the text. The entropy density of radiation at temperature $T$ is $s=(4 / 3) \rho / T=\left(2 \pi^{2} / 45\right) g_{*} T^{3}=1.01 g_{*}^{1 / 4} \rho^{3 / 4}$, where $g_{*}(T)$ is the effective number of particle species in thermal equilibrium, and $\rho=\left(\pi^{2} / 30\right) g_{*} T^{4}$ is the energy density. As the Universe expands the scale factor $a$ increases. The energy density in relativistic particles (radiation) is proportional to $a^{-4}$ and that in non-relativistic particles is proportional to $a^{-3}$. In thermal equilibrium the entropy $a^{3} s$ in a comoving volume is constant and so is $g_{*}^{1 / 3} a T$. According to the Standard Model, $g_{*}^{1 / 4}$ is in the range 1 to 2 for $T \lesssim 100 \mathrm{MeV}$, and then rises sharply to become $\simeq 3$, finally rising to $\simeq 4$ when $T \gtrsim 10^{3}$ GeV in supersymmetric extensions of the Standard Model. We use the appropriate value in our estimates.
} 
As has been discussed recently in connection with ordinary inflation, the assumption that each flaton particle decays independently need not be correct [37 41] (see also [42,8]). Instead, parametric resonance effects can drain away much of the oscillation energy as soon as the oscillation starts, leaving behind only some fraction to decay at the single particle decay rate. The energy drained away goes initially into the creation of marginally relativistic scalar particles. All species are produced which have sufficient coupling to the flaton, including the flaton itself. (We are not aware of any discussion of the possibility of the production of bosons with spin 1 or higher through parametric resonance and it may be that this also occurs. Fermions are not produced in significant number because of Pauli blocking.) If nothing happens to the produced scalar particles they will become non-relativistic after a few Hubble times, and are expected to decay at their one-particle decay rate. 8 If, on the other hand, they thermalize then they turn into highly relativistic radiation.

At the present time it is not clear whether parametric resonance can really create particles which thermalize successfully. However, it is clear that the flaton component of the produced particles cannot thermalize because here one knows that the interaction is too weak. Furthermore, one expects that the energy density of the produced flatons will be a significant fraction of the total energy density [43]. Thus, even if the other produced particles thermalize promptly one expects that a significant fraction of non-thermalized energy will remain, and that a significant fraction of that energy will be in flaton particles.

Any thermalized radiation produced by parametric resonance will redshift away, so independently of the details one expects that a few Hubble times after the end of thermal inflation the energy density is dominated by non-relativistic scalar particles, including the flatons and perhaps other species. Each species will decay at the single-particle decay rate, so we expect eventually to find only the longest-lived species, which dominates the energy density until it decays.

For simplicity we shall assume in what follows that this species is the flaton itself, and we shall also ignore the effect of any radiation produced by particle decay. Thus we are in effect assuming that soon after thermal inflation has ended, some fraction $\epsilon$ of the energy is in non-relativistic flaton particles which decay according to the one-particle decay rate, with the remainder in thermalized radiation. This should describe the real situation at least approximately, provided that any non-flaton particles produced decay at least as rapidly as the flatons. The important special case $\epsilon=1$ is considered, and the possibility that $\epsilon$ may be very small is not discounted. However, as as discussed above, this latter case seems unlikely because one expects that parametric resonance will convert a significant fraction of the energy density into flaton particles which interact too weakly to thermalize.

The upshot of this discussion is that despite the possible occurrence of parametric resonance, one expects that the eventual reheat temperature after thermal inflation is still the temperature $T_{\mathrm{D}}$ calculated from the single-particle decay rate, as given by Eq. (19). If $T_{\mathrm{D}}$ is indeed the reheat temperature, the requirement that it be not too low places strong restrictions on $M$. In order not to upset nucleosynthesis one must have $T_{\mathrm{D}} \gtrsim 10 \mathrm{MeV}$, which requires $M \lesssim 10^{14} \mathrm{GeV}$ (taking $m<10^{3} \mathrm{GeV}$ ). P $^{9}$ However, if $R$ parity is respected as is usually supposed, there is a stable LSP which imposes a much stronger constraint. Indeed, to bring the LSP into thermal equilibrium so that it is not over-produced (and can naturally have the correct abundance to be the dark matter), one needs $T_{\mathrm{D}}$ substantially in excess of the LSP decoupling temperature which is of order $1 \mathrm{GeV}$. Thus one needs $M \lesssim 10^{12} \mathrm{GeV}$. Finally, one might wish to generate baryon number through the electroweak transition which would require $T_{\mathrm{D}} \gtrsim 100 \mathrm{GeV}$ corresponding to $M \lesssim 10^{10} \mathrm{GeV}$. In view of the fact that these limits are perhaps rather conservative (since one expects $\gamma$ to be significantly less than 1 , and does not anticipate $m$ as high as $10^{3} \mathrm{GeV}$ ) this last requirement is hardly likely to be satisfied, but other baryogenesis mechanisms exist as discussed in [13].

\footnotetext{
${ }^{8}$ When the the particles have become non-relativistic one might think that parametric resonance will recommence, since the wavenumber of the corresponding scalar field is negligible compared with its frequency. However, the collection of nonrelativistic particles corresponds to a superposition of almost-classical quantum states, not to any one such state, since the phases of the corresponding fields are uncorrelated, so it is not clear that the parametric resonance formalism applies. More importantly, the amplitude of the would-be classical oscillation will typically be too small for parametric resonance to occur. We are indebted to A. D. Linde for helpful correspondence about this issue.

${ }^{9} \mathrm{In}\left[11\right.$ we estimated $M \lesssim 10^{16} \mathrm{GeV}$. The extra factor 100 came from three different sources. First we used the very naive estimate $\Gamma \sim m^{3} / M^{2}$, corresponding to $\gamma^{1 / 2}=10$. Second, we set $g_{*}^{1 / 2}=1$ where as the true value is more like $10^{1 / 2}$. Third, we rounded up our estimate of $T_{\mathrm{D}}$ to the nearest power of ten which meant multiplying it by of order $10^{1 / 2}$. It so happened that each of these approximations went the same way to give the factor 100 .
} 


\section{COSMOLOGY WITH THERMAL INFLATION}

We now give a systematic account of the history of the early Universe in the case where there is thermal inflation. We assume that there is a moduli problem because this provides the strongest motivation for thermal inflation, and assume that at least some of the moduli have a vev of order $M_{\mathrm{Pl}}$. We also assume that any radiation produced by parametric resonance promptly thermalizes. With these assumptions there are the following eras which we shall consider in turn.

1. Ordinary inflation.

2. Matter domination by the homogeneous oscillation of the inflaton (unless full reheating occurs promptly).

3. Full reheating, which leads to radiation domination if it occurs before the moduli start to oscillate.

4. Homogeneous oscillation of the moduli, starting at the epoch $H \sim m_{\Phi}$. If reheating has previously occurred there is now matter domination by the moduli. If it has not occurred the moduli and inflaton matter densities are roughly comparable, and remain so until full reheating (of the inflaton matter). We assume that full reheating takes place before the beginning of thermal inflation.

5. Thermal inflation.

6. Matter domination by the homogeneous oscillation of the flaton field which caused thermal inflation (unless reheating occurs promptly).

7. Full reheating of the flaton matter, leading to radiation domination before nucleosynthesis after which the history of the Universe is the standard one.

\section{A. Before thermal inflation}

One expects the Universe to start with an era of ordinary inflation [22,23], whether or not there is a later epoch of thermal inflation. During this era, the energy density $\rho$ is dominated by the potential $V$ of the scalar fields, with all except the inflaton field (or fields) fixed. The inflaton field slowly rolls down the potential, because in its direction the flatness conditions $\left|M_{\mathrm{Pl}} V^{\prime} / V\right| \ll 1$ and $\left|V^{\prime \prime}\right| \ll H^{2}$ are satisfied 22, 23]. We noted earlier that in the context of supergravity the second of these conditions requires cancellations. Although these might be accidental it is attractive to suppose that they occur by virtue of some symmetry. One suitable symmetry (most easily implemented in the context of hybrid inflation [25) was suggested in [26,44,45] and another has been proposed in 46. A third possibility is to invoke a global $U(1)$ symmetry as in [47, but this is problematical because the inflaton potential vanishes in the limit where the symmetry is exact so that the magnitude of $V^{\prime \prime}$ is difficult to control ${ }^{10}$ 24

To avoid generating too much large scale cmb anisotropy the potential at the end of ordinary inflation must satisfy

$$
V^{1 / 4} \lesssim 10^{16} \mathrm{GeV}
$$

At some epoch after ordinary inflation 'reheating' occurs, which by definition means that practically all of the energy density thermalizes (except for the contribution of moduli). If reheating is prompt the reheat temperature is $T_{\mathrm{R}} \sim\left(V / g_{*}\right)^{1 / 4}$. A naive estimate of the time taken for reheat would be that it is the decay time of a single inflaton particle, which typically leads to a much lower reheat temperature. However prompt conversion of a large fraction of the energy density into marginally relativistic particles is likely. In the commonly discussed case where inflation ends with the oscillation of a homogeneous inflaton field this is expected to occur through the parametric resonance effect that we considered already for the case of thermal inflation. It is also expected to occur in the case of hybrid inflation though a quantitative account of this case has not yet been given, and will be more complicated because

\footnotetext{
${ }^{10}$ An alternative idea [48] is to suppose that the potential is exactly flat (or at least much flatter than that of the inflaton field) in the direction of at least one field, say a modulus, which couples to the inflaton. The inflaton potential then depends on the value of this field, which will vary from place to place in the Universe allowing the possibility that we live in a region where the inflaton potential happens to be sufficiently flat. But this just pushes back to another level the problem of finding cancellations which keep the potential flat in some direction.
} 
spatial gradients are probably important from the beginning [26]. As we discussed earlier these marginally relativistic particles may then thermalize promptly leading to full or partial reheating.11

Moduli (more precisely, those moduli if any which are flatons with $M \sim M_{\mathrm{Pl}}$ ) are produced both before and after thermal inflation, and we shall call the moduli from these sources respectively big bang moduli and thermal inflation moduli.

When $H \gg m_{\Phi}$ the modulus' potential is given by Eq. (17), so that $\Phi$ is shifted from its true vacuum value by $\Phi_{0}=\Phi_{2}-\Phi_{1} \sim M_{\mathrm{Pl}}$. $\Phi_{2}$ will depend on the composition of the Universe and so $\Phi_{0}$ will change at any phase transitions, such as the end of inflation, but $\Phi$ will rapidly settle down to its new minimum as it is critically damped. However, at the epoch $H \sim m_{\Phi}$ it starts to oscillate about the minimum of its low energy effective potential, and after $H$ has fallen significantly below $m_{\Phi}$, the oscillations will no longer be critically damped and so are much more dangerous.

During thermal inflation $H \ll m_{\Phi}$, so the effective potential is dominated by $V_{\text {true }}$ but $V_{\text {cosm }}$ still gives a small contribution, so the position of the minimum is shifted slightly from the true vacuum value. Oversimplifying a bit, we can estimate the shift by adding together $V_{\text {true }}$ and $V_{\text {cosm }}$ which gives

$$
\begin{aligned}
V & =\frac{1}{2} m_{\Phi}^{2}\left(\Phi-\Phi_{1}\right)^{2}+\frac{\alpha}{2} H^{2}\left(\Phi-\Phi_{2}\right)^{2}+\ldots \\
& =\frac{1}{2} m_{\Phi}^{2} \delta \Phi^{2}+\frac{\alpha}{2} H^{2}\left(\delta \Phi-\Phi_{0}\right)^{2}+\ldots \\
& =\frac{1}{2}\left(m_{\Phi}^{2}+\alpha H^{2}\right)\left(\delta \Phi-\frac{\alpha H^{2}}{m_{\Phi}^{2}+\alpha H^{2}} \Phi_{0}\right)^{2}+\ldots
\end{aligned}
$$

where $\delta \Phi=\Phi-\Phi_{1}$ is the displacement of $\Phi$ from its vev. In the last line $\alpha$ is of order 1 , so the minimum of the modulus' potential is shifted during thermal inflation by an amount of order $\left(H / m_{\Phi}\right)^{2} M_{\mathrm{Pl}}$ [1].

To estimate roughly the abundance of big bang moduli, we can assume that the modulus field starts to oscillate about its vev when $H \sim m_{\Phi}$ with amplitude of order $\Phi_{0} \sim M_{\mathrm{Pl}}$. The energy density $\rho_{\Phi} \sim m_{\Phi}^{2} \Phi_{0}^{2} / 2$ is of order the total energy density. If reheating has already occurred one can crudely set the radiation energy density equal to the total energy density which leads to the estimate

$$
\frac{n_{\Phi}}{s} \sim \frac{\Phi_{0}^{2}}{10 M_{\mathrm{Pl}}^{3 / 2} m_{\Phi}^{1 / 2}}
$$

(In this expression $s$ is the entropy density, and we are using the standard results summarized in the footnote after Eq. (19).) If reheating occurs later the moduli energy density is a fixed fraction of the total until reheating, and again setting the radiation density equal to the total density after reheating one finds

$$
\frac{n_{\Phi}}{s} \sim \frac{\Phi_{0}^{2} H_{\mathrm{R}}^{1 / 2}}{10 M_{\mathrm{Pl}}^{3 / 2} m_{\Phi}}
$$

It is described in the Appendix how a more sophisticated calculation leads to the same results.

We shall assume that full reheating occurs before the onset of thermal inflation (except for the contribution of moduli). The opposite case will be discussed in [13].

These estimates for the moduli apply to any flaton not giving rise to thermal inflation (option (iv) of Section 2.4), if $\Phi_{0}$ is replaced by $M$.

\section{B. Thermal Inflation}

Thermal inflation will occur if one or more of the flaton fields is trapped at the origin in the early Universe. For the moment we suppose that only one is trapped.

\footnotetext{
${ }^{11}$ Note, though, that an extremely low fraction of the energy density cannot thermalize because thermalization requires that the interaction rate per particle exceeds $H$. If the decay products are charged under some gauge symmetry, this requires $\alpha g_{*} T \gtrsim H$ where $\alpha$ is the gauge coupling. Setting $\alpha \sim g_{*}^{-1 / 2} \sim 10^{-1}$, one finds that it is satisfied only if the fraction is bigger than $V /\left(10^{16} \mathrm{GeV}\right)^{4}$. This constraint does not seem to have been noted before in the literature.
} 
The trapping may initially be due to a non-thermal contribution to the mass-squared such as that of order $H^{2}$. However if full reheating occurs before the beginning of thermal inflation then well within a Hubble time of the end of inflation enough entropy to trap the flaton at zero will have been released even by the single particle decay of the inflaton.

If full reheating is indeed delayed to the epoch when thermal inflation begins, the temperature at that epoch is of order $g_{*}^{-1 / 4} V_{0}^{1 / 4} \sim(m M)^{1 / 2}$ corresponding to

$$
\left(\frac{T}{10^{6} \mathrm{GeV}}\right) \sim\left(\frac{M}{10^{10} \mathrm{GeV}}\right)^{1 / 2}
$$

At the other extreme where reheating occurs before the moduli start to oscillate, the temperature at the beginning of thermal inflation is reduced by a factor $\left(M / M_{\mathrm{Pl}}\right)^{1 / 6}$. During thermal inflation $T \propto \exp (-H t)$ and it ends at $T=T_{\mathrm{C}} \sim m$, so there are at most of order $\frac{1}{2} \ln (M / m) \sim 10$ e-folds of thermal inflation. This will not much affect the cosmological density perturbation generated about 50 e-folds before the beginning of ordinary inflation, though there might be a slight change in the spectral index.

\section{Entropy production after thermal inflation}

After thermal inflation ends, relic radiation from the first hot big bang plays no further role. The flaton field now starts to oscillate around its vev with initial amplitude $M$, corresponding to non-relativistic flatons (matter) which dominate the energy density.

The decay of the flaton field generates entropy. If there is no parametric resonance the entropy per comoving volume increases linearly from the end of thermal inflation until the flaton decays, leading to an increase in the entropy by a factor

$$
\Delta \sim \frac{4 V_{0} / 3 T_{\mathrm{D}}}{\left(2 \pi^{2} / 45\right) g_{*}\left(T_{\mathrm{C}}\right) T_{\mathrm{C}}^{3}} \sim \frac{V_{0}}{75 T_{\mathrm{D}} T_{\mathrm{C}}^{3}}
$$

Now suppose instead that there is parametric resonance which promptly thermalizes a substantial fraction of the energy density, leaving a fraction $\epsilon$ in the flatons. This will increase the entropy by a factor

$$
\begin{aligned}
\Delta_{\mathrm{PR}} & \sim \frac{g_{*}\left(T_{\mathrm{PR}}\right)^{1 / 4}(1-\epsilon)^{3 / 4} V_{0}^{3 / 4}}{\left(2 \pi^{2} / 45\right) g_{*}\left(T_{\mathrm{C}}\right) T_{\mathrm{C}}^{3}} \sim \frac{(1-\epsilon)^{3 / 4} V_{0}^{3 / 4}}{25 T_{\mathrm{C}}^{3}} \\
& \sim 10^{10}\left(\frac{M}{10^{10} \mathrm{GeV}}\right)^{\frac{3}{2}}\left(\frac{m}{T_{\mathrm{C}}}\right)^{3}\left(\frac{300 \mathrm{GeV}}{m}\right)^{\frac{3}{2}}\left(\frac{V_{0}}{m^{2} M^{2}}\right)^{\frac{3}{4}}
\end{aligned}
$$

The radiation energy density may initially dominate, but we assume that it falls below that of the residual flatons before the epoch $T_{\mathrm{D}}$. The entropy release from the decay of these flatons is significant only during the era $T_{\mathrm{D}} \lesssim$ $T \lesssim\left(T_{\mathrm{D}}^{4} T_{\text {eq }}\right)^{1 / 5}$ [23], so it is a good approximation to regard this entropy release as suddenly occurring at the epoch $T \sim T_{\mathrm{D}}$. It increases the entropy by a further factor

$$
\begin{aligned}
\Delta_{\mathrm{D}} & \sim \frac{4 \epsilon V_{0} / 3 T_{\mathrm{D}}}{g_{*}\left(T_{\mathrm{PR}}\right)^{1 / 4}(1-\epsilon)^{3 / 4} V_{0}^{3 / 4}} \sim \frac{\epsilon V_{0}^{1 / 4}}{3(1-\epsilon)^{3 / 4} T_{\mathrm{D}}} \\
& \sim 10^{6} \epsilon\left(\frac{M}{10^{10} \mathrm{GeV}}\right)^{\frac{1}{2}}\left(\frac{\mathrm{GeV}}{T_{\mathrm{D}}}\right)\left(\frac{m}{300 \mathrm{GeV}}\right)^{\frac{1}{2}}\left(\frac{V_{0}}{m^{2} M^{2}}\right)^{\frac{1}{4}}
\end{aligned}
$$

The total entropy increase is

$$
\begin{aligned}
\Delta & \sim \Delta_{\mathrm{PR}} \Delta_{\mathrm{D}} \sim \frac{\epsilon V_{0}}{75 T_{\mathrm{D}} T_{\mathrm{C}}^{3}} \\
& \sim 10^{15.5} \epsilon\left(\frac{M}{10^{10} \mathrm{GeV}}\right)^{2}\left(\frac{\mathrm{GeV}}{T_{\mathrm{D}}}\right)\left(\frac{m}{T_{\mathrm{C}}}\right)^{3}\left(\frac{300 \mathrm{GeV}}{m}\right)\left(\frac{V_{0}}{m^{2} M^{2}}\right)
\end{aligned}
$$

Eq. (30) is only supposed to apply if it gives a value $\Delta_{\mathrm{D}}$ bigger than 1 , which fails to be true in the small $\epsilon$ regime $\epsilon \lesssim T_{\mathrm{D}} / V_{0}^{1 / 4}$. This is the regime in which the flaton oscillation fails to dominate the energy density before it disappears at the epoch $T=T_{\mathrm{D}}$. In it $\Delta_{\mathrm{D}}$ is practically equal to 1 , and $\Delta=\Delta_{\mathrm{PR}}$ has the $\epsilon$-independent value given by Eq. (28).

We shall not consider the case where parametric resonance creates radiation which fails to thermalize, and hence quickly reverts to matter in the form of homogeneously oscillating scalar fields. 


\section{Solving the moduli problem with single thermal inflation}

In order not to upset nucleosynthesis, the moduli abundance $n_{\Phi} / s$ must be less than $10^{-12}$ to $10^{-15}$ when nucleosynthesis begins 49. Let us see what is required to satisfy this bound, first for the big bang moduli and then for the moduli produced after thermal inflation.

We can assume that the flaton oscillation comes to dominate the energy density, because the assumption can be shown to be valid in the regime of parameter space satisfying the nucleosynthesis bound on the moduli abundance and to lead to an overestimate of the moduli abundance outside this regime. As a result we can use Eq. (32), and combining it with Eq. 25) one finds that the abundance of big bang moduli after thermal inflation is

$$
\begin{aligned}
\frac{n_{\Phi}}{s} \sim & \frac{\Phi_{0}^{2} H_{\mathrm{R}}^{1 / 2}}{10 m_{\Phi} \Delta M_{\mathrm{Pl}}^{3 / 2}} \sim \frac{8 \Phi_{0}^{2} H_{\mathrm{R}}^{1 / 2} T_{\mathrm{D}} T_{\mathrm{C}}^{3}}{\epsilon m_{\Phi} V_{0} M_{\mathrm{Pl}}^{3 / 2}} \\
\sim & 10^{-16}\left(\frac{10^{12} \mathrm{GeV}}{M}\right)^{\frac{3}{2}}\left(\frac{1}{\epsilon}\right)\left(\frac{M_{\mathrm{Pl}} H_{\mathrm{R}}}{m_{\Phi} M}\right)^{\frac{1}{2}}\left(\frac{T_{\mathrm{D}}}{\mathrm{GeV}}\right) \\
& \times\left(\frac{T_{\mathrm{C}}}{m_{\Phi}}\right)^{3}\left(\frac{\Phi_{0}}{M_{\mathrm{Pl}}}\right)^{2}\left(\frac{m_{\Phi}}{300 \mathrm{GeV}}\right)^{\frac{1}{2}}\left(\frac{m_{\Phi}^{2} M^{2}}{V_{0}}\right)
\end{aligned}
$$

In these formulas $H_{\mathrm{R}}$ is to be considered as being in the range $m_{\Phi}\left(M / M_{\mathrm{Pl}}\right) \lesssim H_{\mathrm{R}} \lesssim m_{\Phi}$. The lower limit comes from our assumption that full reheating after ordinary inflation occurs before the beginning of thermal inflation, and if $H_{\mathrm{R}}$ actually exceeds the upper limit the above formulas give the correct result when it is set equal to this limit.

To analyze these constraints, assume first that $T_{\mathrm{D}} \gtrsim 1 \mathrm{GeV}$ as is required if the LSP is stable, and recall that this implies $M \lesssim 10^{12} \mathrm{GeV}$, from Eq. (19). In Eq. (35), the round brackets in the second line are all of order unity, so we see that the big bang moduli may be sufficiently diluted for $M$ as low as $10^{9} \mathrm{GeV}$, though this requires all parameters to be pushed to the limit and a more reasonable estimate of the lower limit might be $10^{11} \mathrm{GeV}$. Now assume only that $T_{\mathrm{D}} \gtrsim 10 \mathrm{MeV}$, as required by nucleosynthesis, which implies $M \lesssim 10^{14} \mathrm{GeV}$. Then we see that unless $\epsilon$ is very small it should be possible to solve the moduli problem, with no significant additional constraint on $M$.

Now consider the moduli produced after thermal inflation. From Eq. (23), the minimum of the potential during thermal inflation is displaced from its true vacuum minimum by an amount $\delta \Phi \sim\left(V_{0} / m_{\Phi}^{2} M_{\mathrm{Pl}}^{2}\right) \Phi_{0}$. The dynamics at the end of thermal inflation will be complicated but one would expect to generate a moduli number density $n_{\Phi} \sim m_{\Phi} \delta \Phi^{2} / 2 \sim \Phi_{0}^{2} V_{0}^{2} / 2 m_{\Phi}^{3} M_{\mathrm{Pl}}^{4}$ at the end of thermal inflation. Therefore the abundance of thermal inflation moduli is expected to be

$$
\begin{aligned}
\frac{n_{\Phi}}{s} \sim & \frac{\Phi_{0}^{2} V_{0}^{2} / 2 m_{\Phi}^{3} M_{\mathrm{Pl}}^{4}}{g_{*}\left(T_{\mathrm{PR}}\right)^{1 / 4}(1-\epsilon)^{3 / 4} V_{0}^{3 / 4} \Delta_{\mathrm{D}}} \sim \frac{\Phi_{0}^{2} V_{0} T_{\mathrm{D}}}{3 \epsilon m_{\Phi}^{3} M_{\mathrm{Pl}}^{4}} \\
\sim & 10^{-15.5}\left(\frac{M}{10^{12} \mathrm{GeV}}\right)^{2}\left(\frac{1}{\epsilon}\right)\left(\frac{T_{\mathrm{D}}}{\mathrm{GeV}}\right) \\
& \times\left(\frac{\Phi_{0}}{M_{\mathrm{Pl}}}\right)^{2}\left(\frac{300 \mathrm{GeV}}{m_{\Phi}}\right)\left(\frac{V_{0}}{m_{\Phi}^{2} M^{2}}\right)
\end{aligned}
$$

Bearing in mind the relation between $T_{\mathrm{D}}$ and $M$, we see that the abundance of thermal moduli does not impose a significant additional constraint.

Moduli will be produced in the flaton's decay with abundance

$$
\frac{n_{\Phi}}{s} \sim \frac{\Gamma_{\phi \rightarrow \Phi}}{\Gamma} \frac{n_{\phi}}{s}
$$

Since the flaton energy density is $m n_{\phi}$ and we are assuming that it all thermalizes, $n_{\phi} / s$ is of order $T_{\mathrm{D}} / m$ and therefore

$$
\frac{n_{\Phi}}{s} \sim 10^{-16}\left(\frac{\mathrm{GeV}}{T_{\mathrm{D}}}\right)\left(\frac{\Gamma_{\phi \rightarrow \Phi}}{m^{3} / 8 \pi M_{\mathrm{Pl}}^{2}}\right)\left(\frac{m}{300 \mathrm{GeV}}\right)^{2}
$$

which is probably sufficiently small.

Finally we consider the possible thermal creation of gravitinos, moduli and modulinos after thermal inflation. Gravitinos, for which the most detailed calculations exist, appear to be created in a cosmologically safe abundance provided that the maximum temperature is less than [50] $10^{9} \mathrm{GeV}$, and a similar result presumably holds for moduli and modulini since in all cases the interaction with other particles is of gravitational strength. This bound is satisfied after thermal inflation even in the extreme case where most of the energy density thermalizes immediately. 


\section{E. Double Thermal Inflation}

So far we assumed that only one flaton field gives thermal inflation, or in other words that only one flaton field has a thermal mass-squared which traps it at the origin in the early Universe. If two or more flaton fields are trapped the situation is in general much more complicated, but it simplifies considerably if the fields do not interact significantly. We treat this simple situation now, leaving the case of interacting fields to future publications 12, 13]. Thus we consider two flaton fields $\phi_{1}$ and $\phi_{2}$, and assume that each of their potentials is of the form Eq. (2),

$$
V\left(\phi_{1}, \phi_{2}\right)=V_{1}+V_{2}-m_{1}^{2}\left|\phi_{1}\right|^{2}-m_{2}^{2}\left|\phi_{2}\right|^{2}
$$

The higher order terms stabilize the fields at $\phi_{i}=M_{i}$, and the constants $V_{1}$ and $V_{2}$ are the values of the separate potentials at the origin, with $V_{i} \sim m_{i}^{2} M_{i}^{2}$. The critical temperatures at which the fields roll away from zero are $T_{\mathrm{C} i}$, and we take $T_{\mathrm{C} 1}>T_{\mathrm{C} 2}$. When the temperature drops below $T_{\mathrm{C} 1}, \phi_{1}$ will roll away from zero.

If parametric resonance does not produce significant thermalization, the second field now also rolls away promptly and the situation is not substantially different from the case of thermal inflation. If on the other hand a significant fraction of the energy density is thermalized by parametric resonance, the temperature will be raised sufficiently to trap the second field before it has a chance to roll away, leading to a second epoch of thermal inflation driven by the potential

$$
V=V_{2}-m_{2}^{2}\left|\phi_{2}\right|^{2}+\ldots
$$

The residual flatons left after parametric resonance from the first epoch of thermal inflation may be troublesome if they do not decay before nucleosynthesis. Their abundance evaluated after the second epoch of thermal inflation is

$$
\begin{aligned}
\frac{n_{\phi_{1}}}{s} \sim & \frac{\epsilon_{1} V_{1} / m_{\phi_{1}}}{g_{*}\left(T_{\mathrm{PR} 1}\right)^{1 / 4} V_{1}^{3 / 4} \Delta_{2}} \sim \frac{20 \epsilon_{1} V_{1}^{1 / 4} T_{\mathrm{D} 2} T_{\mathrm{C} 2}^{3}}{\epsilon_{2} m_{\phi_{1}} V_{2}} \\
\sim & 10^{-14.5}\left(\frac{M_{1}}{10^{14} \mathrm{GeV}}\right)^{\frac{1}{2}}\left(\frac{10^{12} \mathrm{GeV}}{M_{2}}\right)^{2}\left(\frac{\epsilon_{1}}{\epsilon_{2}}\right)\left(\frac{T_{\mathrm{D} 2}}{\mathrm{GeV}}\right) \\
& \times\left(\frac{T_{\mathrm{C} 2}}{m_{\phi_{1}}}\right)^{3}\left(\frac{m_{\phi_{1}}}{300 \mathrm{GeV}}\right)^{\frac{1}{2}}\left(\frac{V_{1}}{m_{\phi_{1}}^{2} M_{1}^{2}}\right)^{\frac{1}{4}}\left(\frac{m_{\phi_{1}}^{2} M_{2}^{2}}{V_{2}}\right)
\end{aligned}
$$

Thus, a second epoch of thermal inflation may significantly dilute the residual flatons from a first epoch, which could remove the restriction $M_{1} \lesssim 10^{14} \mathrm{GeV}$ which is otherwise demanded by nucleosynthesis. Conceivably one may in this way make thermal inflation viable with a GUT Higgs field or even with a modulus, though more investigation is needed to see whether this is a real possibility.

Henceforth we will assume that $M_{1}$ is sufficiently small to allow $\phi_{1}$ to decay before nucleosynthesis, which allows us to take $M_{2}$ small enough to have a comfortably high final reheat temperature.

\section{F. Solving the moduli problem with double thermal inflation}

For simplicity we take the abundance of big bang moduli before thermal inflation to be $n_{\Phi} / s \sim 0.1 \Phi_{0}^{2} M_{\mathrm{Pl}}^{-3 / 2} m_{\Phi}^{-1 / 2}$ and assume that parametric resonance leads to effectively complete reheating so that $\epsilon \simeq 0$. These assumptions lead to the maximum possible moduli abundance. Each epoch of thermal inflation then increases the entropy by a factor $\Delta_{i} \sim \Delta_{\mathrm{PR} i} \sim V_{i}^{3 / 4} / 25 T_{\mathrm{C} i}^{3}$. Therefore the abundance of big bang moduli after double thermal inflation is

$$
\begin{aligned}
\frac{n_{\Phi}}{s} \sim & \frac{\Phi_{0}^{2}}{10 m_{\Phi}^{1 / 2} \Delta_{1} \Delta_{2} M_{\mathrm{Pl}}^{3 / 2}} \sim \frac{60 \Phi_{0}^{2} T_{\mathrm{C} 1}^{3} T_{\mathrm{C} 2}^{3}}{m_{\Phi}^{1 / 2} V_{1}^{3 / 4} V_{2}^{3 / 4} M_{\mathrm{Pl}}^{3 / 2}} \\
\sim & 10^{-17.5}\left(\frac{10^{13} \mathrm{GeV}}{M_{1}}\right)^{\frac{3}{2}}\left(\frac{10^{10} \mathrm{GeV}}{M_{2}}\right)^{\frac{3}{2}}\left(\frac{T_{\mathrm{C} 1}}{m_{\Phi}}\right)^{3}\left(\frac{T_{\mathrm{C} 2}}{m_{\Phi}}\right)^{3} \\
& \times\left(\frac{\Phi_{0}}{M_{\mathrm{Pl}}}\right)^{2}\left(\frac{m_{\Phi}}{300 \mathrm{GeV}}\right)^{\frac{5}{2}}\left(\frac{m_{\Phi}^{2} M_{1}^{2}}{V_{1}}\right)^{\frac{3}{4}}\left(\frac{m_{\Phi}^{2} M_{2}^{2}}{V_{2}}\right)^{\frac{3}{4}}
\end{aligned}
$$

The abundance of thermal inflation moduli produced at the end of the first epoch of thermal inflation evaluated after the second epoch of thermal inflation is 


$$
\begin{aligned}
\frac{n_{\Phi}}{s} \sim & \frac{\Phi_{0}^{2} V_{1}^{2} / 2 m_{\Phi}^{3} M_{\mathrm{Pl}}^{4}}{g_{*}\left(T_{\mathrm{PR} 1}\right)^{1 / 4} V_{1}^{3 / 4} \Delta_{2}} \sim \frac{3 \Phi_{0}^{2} V_{1}^{5 / 4} T_{2}^{3}}{m_{\Phi}^{3} V_{2}^{3 / 4} M_{\mathrm{Pl}}^{4}} \\
\sim & 10^{-16.5}\left(\frac{M_{1}}{10^{13} \mathrm{GeV}}\right)^{\frac{5}{2}}\left(\frac{10^{10} \mathrm{GeV}}{M_{2}}\right)^{\frac{3}{2}}\left(\frac{T_{2}}{m_{\Phi}}\right)^{3} \\
& \times\left(\frac{\Phi_{0}}{M_{\mathrm{Pl}}}\right)^{2}\left(\frac{m_{\Phi}}{300 \mathrm{GeV}}\right)\left(\frac{V_{1}}{m_{\Phi}^{2} M_{1}^{2}}\right)^{\frac{5}{4}}\left(\frac{m_{\Phi}^{2} M_{2}^{2}}{V_{2}}\right)^{\frac{3}{4}}
\end{aligned}
$$

The abundance of thermal inflation moduli produced at the end of the second epoch of thermal inflation is

$$
\begin{aligned}
\frac{n_{\Phi}}{s} & \sim \frac{\Phi_{0}^{2} V_{2}^{2} / 2 m_{\Phi}^{3} M_{\mathrm{Pl}}^{4}}{g_{*}\left(T_{\mathrm{PR} 2}\right)^{1 / 4} V_{2}^{3 / 4}} \sim \frac{\Phi_{0}^{2} V_{2}^{5 / 4}}{8 m_{\Phi}^{3} M_{\mathrm{Pl}}^{4}} \\
& \sim 10^{-14}\left(\frac{M_{2}}{10^{10} \mathrm{GeV}}\right)^{\frac{5}{2}}\left(\frac{\Phi_{0}}{M_{\mathrm{Pl}}}\right)^{2}\left(\frac{300 \mathrm{GeV}}{m_{\Phi}}\right)^{\frac{1}{2}}\left(\frac{V_{2}}{m_{\Phi}^{2} M_{2}^{2}}\right)^{\frac{5}{4}}
\end{aligned}
$$

We see that two independent bouts of thermal inflation can solve the moduli problem for a wide range of the vevs, even if parametric resonance is extremely efficient.

\section{G. Topological defects}

We end this paper with a brief discussion of the cosmological production of topological defects, namely walls, strings, monopoles and textures.

Each type of topological defect is associated with a scalar field (in general multi-component) with nonzero vev. Among several possibilities, we consider here only two cases. The first is that the vev belongs to a GUT higgs potential, and that it has the non-flat form usually considered. The second is that the vev belongs to a flat potential.

For a GUT higgs with the standard non-flat potential the temperature after inflation is never high enough for the defects to form by the usual Kibble mechanism [32, 33. (We are not of course concerned with any defects forming before ordinary inflation since they have been diluted away.) They can only form near or at the end of ordinary inflation, and even that requires that the bound Eq. (20) on the inflationary potential is saturated [26].

Consider first monopoles, using the standard results 32]. The abundance of monopoles, after some initial annihilation, settles down soon after the GUT transition to a value $n / s \sim 10^{-10}$. The strongest bound on their present abundance comes from baryon decay catalysis in neutron stars, which requires $n / s \lesssim 10^{-37}$. Thus the entropy must increase by a factor $10^{27}$ between the end of ordinary inflation and the present. If reheating after ordinary inflation is prompt, the factor is the one $\Delta$ arising from thermal inflation. We see from Eq. (33) that a single bout of thermal inflation is probably insufficient, but two bouts could be enough. Alternatively, if reheating after ordinary inflation is long delayed this gives an additional increase $\Delta_{\text {ord }} \sim 10^{16} \mathrm{GeV} / T_{\mathrm{R}}$, which could be enough to make just one bout of thermal inflation viable.

Depending on the GUT symmetry, gauge strings might also be produced, which would be cosmologically significant perhaps providing candidates for the origin of large scale structure. On cosmological scales their evolution is not affected by thermal inflation because their spacing is outside the horizon during that epoch. (This is just the statement that there are much less than $50 e$-folds of thermal inflation.) The same applies to other defect networks formed before thermal inflation (global domain walls, monopoles, strings or textures).

Consider now defects associated with a flat potential. They form if at all at the end of thermal inflation. Consider first the case of $Z_{n}$ symmetry (Sections 2.1 and 2.3). A discrete symmetry used to be regarded as problematical for cosmology, because when it is spontaneously broken it seems to lead to cosmologically forbidden domain walls. However, if the symmetry is also explicitly broken, as will typically be the case for the flaton potential, there need be no problem because walls do not necessarily form and if they do form they do not necessarily survive (because the vacua on either side of a wall may have different energy density). If, on the other hand, it is exact it will probably be a discrete gauge symmetry which again avoids the domain wall problem because there is only one physical vacuum.

If there is a global $U(1)$ symmetry, strings can form at the end of thermal inflation with the strings later joined by walls if the symmetry is approximate. An example of this might be Peccei-Quinn symmetry [12]. Local strings forming at the end of thermal inflation would have too little energy to be cosmologically significant. Finally, if the flaton field giving rise to thermal inflation has two or more components as in Section 3.5 then monopoles or textures might form at the end of thermal inflation but we have not considered this case. 


\section{SUMMARY AND CONCLUSION}

Flatons are scalar fields with masses $m$ of order $10^{2}$ to $10^{3} \mathrm{GeV}$ and vacuum expectation values $M \gg m$. They arise naturally in supersymmetric theories and indeed it is not unreasonable to suppose that they are the only source of vevs in this range (in the observable sector). Flatons with $M \gtrsim 10^{14} \mathrm{GeV}$ are cosmologically dangerous, and in particular moduli with $M \sim M_{\mathrm{Pl}}$ are overproduced by twenty orders of magnitude in the standard cosmology, which is the well-known Polonyi/moduli problem. In this paper we have explained how the problem may be solved by flatons with smaller vevs, in the range $10^{9} \mathrm{GeV} \lesssim M \lesssim 10^{13} \mathrm{GeV}$ that is theoretically very natural for flatons other than moduli.

Such flatons solve the moduli problem by generating an era of thermal inflation. Thermal inflation occurs when the flaton is held at zero by thermal effects, and it typically lasts for about $10 e$-folds and occurs at a very low energy scale. These properties are precisely what is required to sufficiently dilute the moduli produced before thermal inflation without affecting the density perturbation produced during ordinary inflation (10 $e$-folds), while not regenerating them again afterwards (low energy scale). Detailed calculations show that a single epoch of thermal inflation driven by a flaton whose vev is within one or two orders of magnitude of $10^{12} \mathrm{GeV}$ can solve the moduli problem, though the constraints are quite tight.

It is easier for thermal inflation to rescue flatons with vev's $M \gtrsim 10^{14} \mathrm{GeV}$ (in particular, moduli with $M \sim M_{\mathrm{Pl}}$ ) if the latter do not themselves give rise to thermal inflation. Remarkably, segregation of flatons into a class which thermally inflate and have small vevs, and a class which do not and have large vevs is exactly what one expects from a theoretical viewpoint. The larger the vev of a flaton, the less likely it is to be trapped at the origin in the early Universe, because the finite temperature contribution to the effective potential becomes ineffective at field values bigger than the temperature.

There are several aspects of cosmology which we have not addressed in the present paper, notably axion cosmology and baryogenesis which will be the subjects of respectively [12,13. Let us close by briefly discussing the latter topic. As successful thermal inflation sufficiently dilutes any pre-existing moduli it will also dilute any pre-existing baryon number to negligible amounts. However, as will be discussed in [13], there are several possibilities for baryogenesis within the context of thermal inflation itself. One especially promising mechanism can occur if the flaton which gives rise to thermal inflation also generates the mass of a right-handed neutrino. A lepton asymmetry can then be generated after thermal inflation. The partial reheat temperature after thermal inflation can be high enough to restore the electroweak symmetry, and so this lepton asymmetry can be converted into a baryon asymmetry by non-perturbative electroweak effects [51].

\section{APPENDIX}

To arrive at a more sophisticated estimate of the moduli abundance we solve the equation of motion of the modulus in the potential Eq. (22), which is

$$
\ddot{\delta \Phi}+3 H \dot{\delta \Phi}+m_{\Phi}^{2} \delta \Phi+\alpha H^{2}\left(\delta \Phi-\Phi_{0}\right)=0
$$

where we take $H=p / t$ with $p=1 / 2$ for radiation domination and $p=2 / 3$ for matter domination. One would expect $\alpha \sim 1$. An estimate of $\Phi_{0}$ can be obtained by taking the distance between the self-dual points of the target space modular symmetry $\mathrm{SL}(2, \mathbf{Z})$ [52] using the usual orbifold Kähler metric for the moduli. This gives $\Phi_{0}^{2} \sim 0.1$. In this case one can easily check that our approximation of neglecting the contribution of the moduli to the energy density of the Universe before the asymptotic solution is attained is consistent. $\delta \Phi$ will rapidly settle to $\delta \Phi=\Phi_{0}$ when $H \gg m_{\Phi}$, and so we take $\delta \Phi(0)=\Phi_{0}$ and $\dot{\delta \Phi}(0)=0$. With these initial conditions, Eq. (50) has the solution

$$
\delta \Phi=\alpha p^{2} \Phi_{0}\left(\frac{1}{m_{\Phi} t}\right)^{\frac{3 p-1}{2}} s_{\mu, \nu}\left(m_{\Phi} t\right)
$$

where $s_{\mu, \nu}$ is a Lommel function, $\mu=-3(1-p) / 2$ and $\nu^{2}=-\alpha p^{2}+(3 p-1)^{2} / 4$. At late times

$$
\delta \Phi \sim \frac{\alpha \Phi_{0}}{\sqrt{\pi}}\left(\frac{p}{2}\right)^{\frac{4-3 p}{2}} \Gamma\left(\frac{1+\mu}{2}+\frac{\nu}{2}\right) \Gamma\left(\frac{1+\mu}{2}-\frac{\nu}{2}\right)\left(\frac{H}{m_{\Phi}}\right)^{\frac{3 p}{2}} \sin \left(m_{\Phi} t+\frac{(2-3 p) \pi}{4}\right)
$$

The coefficient has a weak dependence on $\alpha$ and $p$, and for $\alpha \sim 1$ and $p=1 / 2$ or $2 / 3$ we get to a good approximation 


$$
\delta \Phi \sim \frac{4}{3} \Phi_{0}\left(\frac{H}{m_{\Phi}}\right)^{\frac{3 p}{2}} \sin \left(m_{\Phi} t+\frac{(2-3 p) \pi}{4}\right)
$$

Therefore the moduli abundance is given by

$$
\frac{n_{\Phi}}{s} \sim \frac{\Phi_{0}^{2}}{10 m_{\Phi}^{1 / 2}}\left(\frac{H}{m_{\Phi}}\right)^{\frac{3(2 p-1)}{2}}
$$

Setting $p=1 / 2$ gives Eq. (24) and setting $p=2 / 3$ gives Eq. (25).

\section{Acknowledgements}

While this work was in progress EDS was supported by a Royal Society Fellowship at Lancaster University. EDS is now supported by the JSPS. DHL acknowledges funding from PPARC and the EC, and both authors acknowledge funding from the Aspen Physics Institute where the work was completed. We are indebted to Andrei Linde for extensive correspondence about parametric resonance, and have benefited from useful discussions with Graham Ross, Beatriz de Carlos, Andre' Lukas, Raghavan Rangarajan, Lisa Randall and Steven Thomas.

[1] K. Yamamoto, Phys. Lett. 161B, 289 (1985).

[2] G. D. Coughlan et al., Phys. Lett. 131B, 59 (1983).

[3] M. Dine, W. Fischler and D. Nemeschansky, Phys. Lett. 136B, 169 (1984).

[4] G. D. Coughlan, R. Holman, P. Ramond and G. G. Ross, Phys. Lett. 140B, 44 (1984).

[5] K. Yamamoto, Phys. Lett. 168B, 341 (1986).

[6] G. Lazarides, C. Panagiotakopoulos and Q. Shafi, Phys. Rev. Lett. 56, 557 (1986).

[7] K. Enqvist, D. V. Nanopoulos and M. Quiros, Phys. Lett. 169B, 343 (1986).

[8] O. Bertolami and G. G. Ross, Phys. Lett. B183, 163 (1987).

[9] K. Yamamoto, Phys. Lett. B194, 390 (1987).

[10] J. Ellis, K. Enqvist, D. V. Nanopoulos and K. A. Olive, Phys. Lett. B188, 415 (1987); G. Lazarides, C. Panagiotakopoulos and Q. Shafi, Nucl. Phys. B307, 937 (1988); J. Ellis, K. Enqvist, D. V. Nanopoulos and K. A. Olive, Phys. Lett. B225, 313 (1989).

[11] D. H. Lyth and E. D. Stewart, Phys. Rev. Lett. 75, 201 (1995).

$[12]$ D. H. Lyth, in preparation.

[13] E. D. Stewart, in preparation.

[14] For reviews of supersymmetry, see H. P. Nilles, Phys. Rep. 110, 1 (1984) and D. Bailin and A. Love, Supersymmetric Gauge Field Theory and String Theory, IOP, Bristol (1994).

[15] J. Polonyi, Budapest preprint KFKI-1977-93, unpublished (1977).

[16] J. Ellis, D. V. Nanopoulos and M. Quiros, Phys. Lett. B174, 176 (1986); B. de Carlos, J. A. Casas, F. Quevedo and E. Roulet, Phys. Lett. B318, 447 (1993).

[17] T. Banks, D. B. Kaplan and A. E. Nelson, Phys. Rev. D49, 779 (1994).

[18] L. Randall and S. Thomas, Nucl. Phys. B449, 229 (1995).

[19] T. Banks, M. Berkooz and P. J. Steinhardt, Phys. Rev. D52, 705 (1995).

[20] M. Dine, L. Randall and S. Thomas, Phys. Rev. Lett. 75, 398 (1995).

[21] H. Murayama, H. Suzuki and T. Yanagida, Phys. Lett. B291, 418 (1992).

[22] A. D. Linde, Particle Physics and Inflationary Cosmology, Harwood Academic, Switzerland (1990).

[23] E. W. Kolb and M. S. Turner, The Early Universe, Addison-Wesley, New York (1990).

[24] D. H. Lyth, Phys. Lett. 147B, 403 (1984); 150B, 465(E) (1985); A. R. Liddle, Phys. Rev. D49, 739 (1994).

[25] A. D. Linde, Phys. Lett. B259, 38 (1991).

[26] E. J. Copeland, A. R. Liddle, D. H. Lyth, E. D. Stewart and D. Wands, Phys. Rev. D49, 6410 (1994).

[27] M. Dine, L. Randall and S. Thomas, hep-ph/9507453.

[28] D.-G. Lee and R. N. Mohapatra, hep-ph/9502210; B. Brahmachari and R. N. Mohapatra, hep-ph/9505347; J. Sato, hepph/9508269; B. Brahmachari and R. N. Mohapatra, hep-ph/9508293.

[29] J E Kim, Phys. Rep. 150, 1 (1987). 
[30] D. H. Lyth, Phys. Rev. D45, 3394 (1992); D. H. Lyth and E. D. Stewart, Phys. Rev. D46, 532 (1992); D. H. Lyth, Phys. Rev. D48, 4523 (1993).

[31] E. J. Chun and A. Lukas, Phys. Lett. B357, 43 (1995).

[32] A. Vilenkin and E. P. S. Shellard, Cosmic Strings and other Topological Defects, Cambridge University Press, Cambridge (1994).

[33] M. B. Hindmarsh and T. W. B. Kibble, hep-ph/9411342, to appear in Phys. Rep..

[34] K. S. Babu and R. N. Mohapatra, Phys. Rev. Lett. 74, 2418 (1995).

[35] T. Barreiro, E. J. Copeland, D. H. Lyth and T. Prokopec, in preparation.

[36] M. Dine et al., Nucl. Phys. B259, 549 (1985); F. del Aguila, G. Blair, M. Daniel and G. G. Ross, Nucl. Phys. B272, 413 (1986); B. R. Greene, K. H. Kirklin, P. J. Miron and G. G. Ross, Nucl. Phys. B292, 606 (1987); G. Dvali and Q. Shafi, Phys. Lett. B339, 241 (1994); G. Aldazabal, A. Font, L. E. Ibáñez and A. M. Uranga, hep-th/9410206; L. J. Hall and S. Raby, Phys. Rev. D51, 6524 (1995); L. E. Ibáñez, hep-th/9505098; G. Aldazabal, A. Font, L. E. Ibáñez and A. M. Uranga, hep-th/9508033.

[37] J. H. Traschen and R. H. Brandenberger, Phys. Rev. D42, 2491 (1990).

[38] L. Kofman, A. D. Linde and A. A. Starobinsky, Phys. Rev. Lett. 73, 3195 (1994); L. Kofman, A. D. Linde and A. A. Starobinsky, hep-th/9510119 (1995).

[39] Y. Shtanov, J. Traschen and R. Brandenberger, Phys. Rev. D51, 5438 (1995).

[40] D. Boyanovsky and H. J. de Vega, Phys. Rev. D47, 2343 (1993); D. Boyanovsky, D.-S. Lee and A. Singh, Phys. Rev. D48, 800 (1993); D. Boyanovsky, H. J. de Vega and R. Holman, Phys. Rev. D49, 2769 (1994); D. Boyanovsky et al., hep-ph/9507414 to appear in Phys. Rev D (1995); D. Boyanovsky et al., hep-ph/9511361 (1995).

[41] M. Yoshimura, hep-th/9506176, Prog. Theor. Phys. 94, in press (1995); D. I. Kaiser, astro-ph/9507108 (1995); H. Fujisaki et al., hep-ph/9508378 (1995); H. Fujisaki et al., hep-ph/9511381 (1995).

[42] B. A. Ovrut and P. J. Steinhardt, Phys. Lett. 147B, 263 (1984).

[43] A. D. Linde, personal communication.

[44] E. D. Stewart, Phys. Rev. D51, 6847 (1995).

[45] E. D. Stewart, Phys. Lett. B345, 414 (1995).

[46] M. K. Gaillard, H. Murayama and K. A. Olive, Phys. Lett. B355, 71 (1995).

[47] K. Freese, J. A. Frieman and A. V. Olinto, Phys. Rev. Lett. 65, 3233 (1990); F. C. Adams et al., Phys. Rev. D47, 426 (1993).

[48] G. G. Ross and S. Sarkar, hep-ph/9506283.

[49] J. Ellis et al., Nucl. Phys. B373, 399 (1992).

[50] R. G. Leigh and R. Rattazzi, Phys. Lett. B352, 20 (1995); J. Ellis, D.V. Nanopoulos, K. A. Olive and S.-J. Rey, hepph/9505438. For a dissenting view see W. Fischler, Phys. Lett. B332, 277 (1994).

[51] M. Fukugita and T. Yanagida, Phys. Lett. B174, 45 (1986).

[52] See for example M. Cvetič et al., Nucl. Phys. B361, 194 (1991). 